\title{
Bare LO-Phonon Peak in THz-Emission Signals: a Dielectric-Function Analysis
}

\author{
Fabrício M. Souza and J. C. Egues* \\ Departamento de Física e Informática, Instituto de Física de São Carlos, \\ Universidade de São Paulo, 13560-970 São Carlos, São Paulo, Brasil
}

Received on 23 April, 2001

\begin{abstract}
We present a normal-mode analysis of coupled photocarrier-phonon dynamics in Te. We consider a dielectric function which accounts for LO phonons and the electron-hole gas within the DebyeHückel model and RPA. Our main finding is the existence of a bare LO phonon mode in the system even at high carrier density. This oscillation is an unscreened $L_{-}(q)$ mode arising from ineffective screening at large wave vectors. This mode is consistent with the bare LO-phonon peak in recent THz-emission spectra of Te.
\end{abstract}

\section{Introduction}

The recent progress in ultrafast spectroscopy allows the investigation of photocarrier-phonon coupled $\mathrm{THz}$ dynamics in semiconductors [1]. Ultrashort pulses enable the excitation and detection of coherent infrared lattice vibrations which is a source of $\mathrm{THz}$ radiation. $\mathrm{THz}$ emission has been measured recently in semiconductors via a dipole antenna with detectable frequencies up to around $5 \mathrm{THz}$ [2-4]. Tellurium is a suitable semiconductor for these experiments because it possesses infraredactive modes within the antenna spectral range.

The physics of $\mathrm{THz}$ emission in Te is straightforward. A laser pulse generates a non-equilibrium distribution of electron-hole pairs. The absorption length at the laser energies used in the experiments $(\sim 1.7 \mathrm{eV})$ is $40 \mathrm{~nm}$ [5]. This small absorption length creates a highly inhomogeneous longitudinal charge density and diffusion currents arise. These currents lead to charge separation, due to the different electron and hole mobilities, which generates a longitudinal polarization field (Dember field). The Dember field drives LO phonons (Te is piezoelectric) whose macroscopic oscillatory polarization emits radiation.

The emitted spectrum from the Te crystal consists of a broad peak at approximately $0.5 \mathrm{THz}$, followed by a dip to approximately zero at $\nu_{T O}=2.6 \mathrm{THz}$ and a second maximum close to $\nu_{L O}=2.82 \mathrm{THz}$ [3],[4]. The peak at $0.5 \mathrm{THz}$ is related to the ultrafast buildup of the Dember field [3]. The bare LO phonon mode is usually explained in terms of the lateral inhomogeneity of the excitation spot. In this picture the low-density "wings" of the spot gives rise to phonon-like long wave- length $(q=0)$ modes at the bare LO frequency [see $L_{+}$ branch in Fig. 1(a)].

Here we use a simple normal-mode analysis to gain further insight into the physics behind the bare LO peak observed in the emission spectrum of Te. Interestingly enough, we find that the longitudinal inhomogeneity of the electron-hole gas gives rise to bare LO phonon modes even at high carrier densities. This is in contrast with the usual picture involving lateral inhomogeneity of $q=0$ modes. We believe longitudinal inhomogenety $(q \neq 0)$ leading to ineffective screening of the phonon polarization fields by the electron-hole gas provides an alternate (or complementary) description for the observed bare LO frequency.

Dielectric function. In a first approximation the total dielectric function of the electron-hole-phonon system is given by

$$
\epsilon(q, \omega)=\epsilon_{\infty}+\frac{\epsilon_{0}-\epsilon_{\infty}}{1-\left(\frac{\omega}{\omega_{T O}}\right)^{2}}-V_{q} P(q, \omega),
$$

with

$$
V_{q} P(q, \omega)=\sum_{i=e, h} \frac{\omega_{p l, i}^{2} \epsilon_{\infty}}{\omega^{2}-\frac{k_{B} T_{i}}{m_{i}^{*}} q^{2}},
$$

where $\omega_{p l, i}=\sqrt{\frac{4 \pi n_{i} e^{2}}{\epsilon_{\infty} m_{i}^{*}}}$ is the plasma frequency for electrons $(i=e)$ or holes $(i=h), m_{i}^{*}$ is the effective mass $\left(m_{e / h}^{*}=0.067 / 0.53 m_{0}\right), n_{i}$ is the particle density, $e$ is the electron charge, $\epsilon_{\infty}=10.9$ is the dielectric constant for frequencies much above the reststrahl $\omega \gg \omega_{T O}=$ $2 \pi \nu_{T O}, \epsilon_{0}=12.8$ is the static dielectric constant, $T_{i}$ is the carrier temperature and $k_{B}$ is the Boltzmann constant. Apart from a $\epsilon_{\infty}$ additive term, Eq. (2) is

\footnotetext{
*email:egues@if.sc.usp.br
} 
essentially the Debye-Hückel dielectric function within a hydrodynamic model [6]. Note that $\epsilon(q, \omega)$ takes into account the longitudinal inhomogeneity of the electronhole gas through the $q$ dependence in (2). Longitudinal inhomogeneity plays a fundamental role in the Te system because it gives rise to a driving Dember field which excites the infrared lattice modes.

Normal modes. The zeros of the dielectric function (1) define the normal-mode frequencies of the system: $\epsilon(q, \omega)=0 \Rightarrow \omega(q, n)$, where $n$ is the carrier density (we assume $n=n_{e}=n_{h}$ ). Fig. 1(a) shows the usual dispersion relation for $q=0$ as a function of the carrier densities [7]. The branches $L_{+}$and $L_{-}$(solid lines) arise from the anti-crossing of LO phonons and plasmons. The important point here is that the $L_{-}$branch goes to the screened LO frequency $\nu_{T O}$ as $n$ increases. This is due to screening of the lattice polarization field by the plasma.

Fig. 1(b) shows the $L_{-}$branch for $n=10^{18} \mathrm{~cm}^{-3}$ as a function of $q$. For $q \rightarrow 0$ we recover the screened LO frequency since $n$ is high. When $q$ increases, however, screening becomes inefficient and the $L_{-}$branch shifts to the bare LO frequency $\nu_{L O}[8]$. Hence screening ineffectiveness is a possible mechanism to generate a bare LO peak in the spectrum of the electron-hole-phonon system.

It is instructive to compare the Debye-Hückel and RPA dielectric function analyses. The RPA dielectric function is given by [9],

$$
V_{q} P(q, \omega)=\frac{4 \pi e^{2}}{q^{2} \Omega} \sum_{i=e, h} \sum_{\mathbf{k}} \frac{f_{i}\left(\xi_{i \mathbf{k}}\right)-f\left(\xi_{i \mathbf{k}+\mathbf{q}}\right)}{\omega-\xi_{i \mathbf{k}}+\xi_{i \mathbf{k}+\mathbf{q}}+i \delta} .
$$

where $\Omega$ is the sample volume, $f_{i}\left(\xi_{i \mathbf{k}}\right)$ is the Fermi distribution function for electrons or holes and $\xi_{i \mathbf{k}}$ is the free electron or hole energy. Fig. 2(b) clearly shows that RPA and Debye-Hückel yield similar dispersion curves for $L_{-}(q)$. This is consistent with the high temperatures used in the calculation $\left(T_{e / h}=7000 / 3600 \mathrm{~K}\right)$ [10]. Note that we are not assuming $q \rightarrow 0$ in the RPA contribution (3).

The main result of our simple normal-mode analysis - namely, that the longitudinal inhomogeneity of the electron-hole gas makes screening ineffective thus allowing for bare LO modes even at high densities also follows from a more detailed numerical investigation [11]. We have solved a set of coupled differential equations consisting of Poisson's equation, a drivenharmonic-oscillator equation and transport equations which include inhomogeneity via diffusion terms. The numerical spectra are similar to the experimental ones, i.e., they also show a broad peak near $0.5 \mathrm{THz}$, a dip at $2.6 \mathrm{THz}$ and a second peak close to $2.82 \mathrm{THz}$ [12].

In conclusion, the dielectric function analysis presented here reveals that non-zero wave vector modes lead to bare LO phonon oscillations of the electronhole-phonon system even at high carrier density. This is due to the suppression of the screening of the lattice polarization field by the inhomogeneous electron-hole gas. This screening ineffectiveness provides an alternative/complementary description for the observed bare LO frequency in $\mathrm{THz}$ emission experiments.

The authors acknowledge support from FAPESP and CAPES.

Present address: University of Basel, Institute of Physics, Klingelbergstrasse 82, CH-4056 Basel, Switzerland.
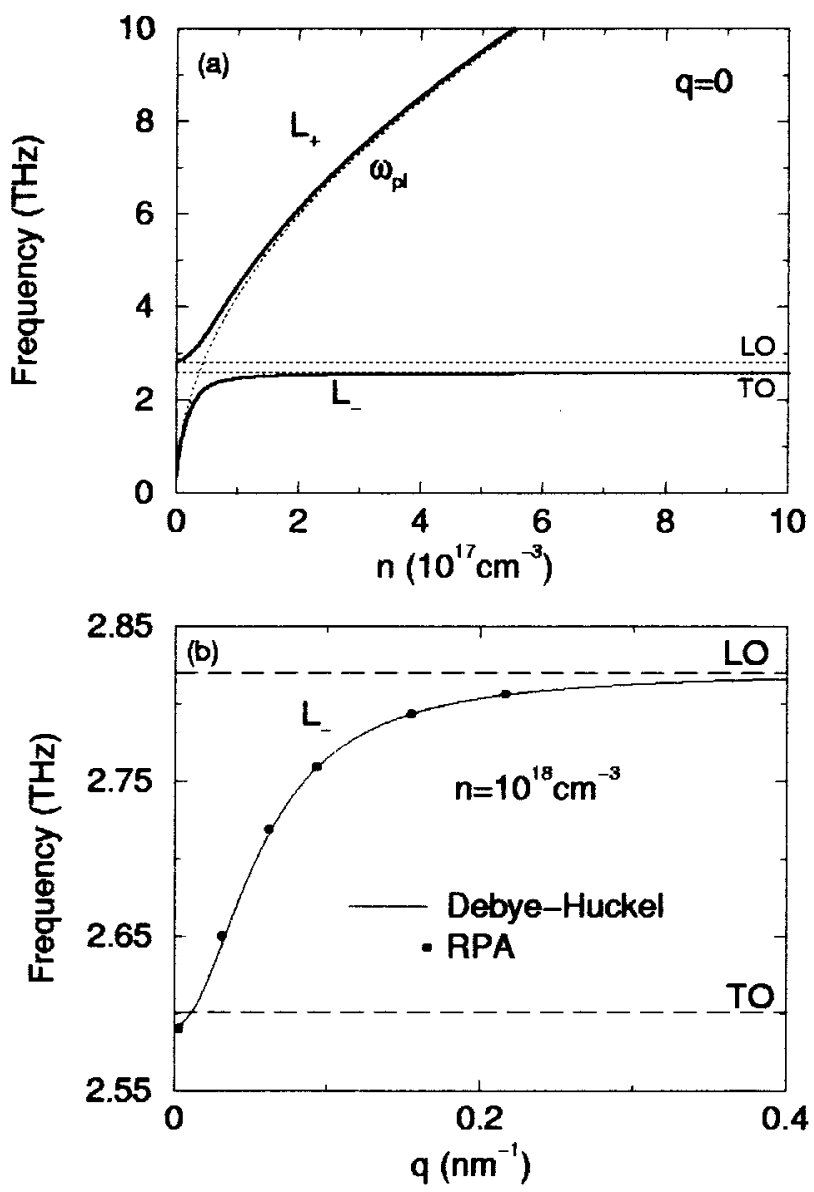

Figure 1. Plasmon-phonon coupled modes for Tellurium as a function of carrier density [for $q=0$ ] (a) and wave vector $q$ (b). The lower branch $L_{-}$(a) tends to the TO frequency value for high densities due to the efficient screening of the lattice polarization field by the electron-hole gas. Ineffective screening on the other hand, relevant for non-zero wave vectors $q(\mathrm{~b})$, makes the $L_{-}$branch move towards the LO frequency limit for increasing $q$ 's - even at high densities. This is in contrast with the $q=0$ mode in (a).

\section{References}

[1] T. Dekorsy et al., Top. Appl. Phys. 76, 169 (2000).

[2] T. Dekorsy et al., Phys. Rev. Lett. 74, 738 (1995). 
[3] T. Dekorsy et al., Phys. Rev. B 53, 4005 (1996).

[4] M. Tani et al., J. Appl. Phys. 83, 2473 (1998).

[5] The Te crystal is hexagonal with three atoms per unit cell arranged in a helix along the crystalographic $c$ axis. The excitation surface considered here is perpendicular to the $c$ axis $\left(c_{\perp}\right.$ geometry).

[6] G. Rickayzen, Green's Functions and Condensed Matter, Academic Press, New York (1991).

[7] A. Mooradian, A. L. Mc Whorter, Phys. Rev. Lett. 19, 849 (1967).

[8] See R. A. Cowley and G. Dolling, Phys. Rev. Lett. 14, 549 (1965) for a similar effect in the context of optical modes of ionic crystals.
[9] G. D. Mahan, Many-Particle Physics, Plenum Press, New York (1990).

[10] To calculate the $k$ sum in Eq.(3) in the hightemperature limit, we use the approach of H. Sato and Y. Hori, Phys. Rev. B 36, 6033 (1987).

[11] A brief account of this study with preliminary results for the coupled dynamics of the electric and phonon fields in GaAs can be found in Fabrício M. Souza and J. C. Egues, Braz. J. Phys. 29, 831 (1999).

[12] Fabrício M. Souza and J. C. Egues, cond-mat/0105529 (submitted). 